\title{
Direct Visualization of the Movement of the Monomeric Axonal Transport Motor UNC-104 along Neuronal Processes in Living Caenorhabditis elegans
}

\author{
H. Mimi Zhou, Ingrid Brust-Mascher, and Jonathan M. Scholey \\ Section of Molecular and Cellular Biology, University of California at Davis, Davis, California 95616
}

The formation and function of axons depends on the microtubule-based transport of cellular components from their sites of synthesis in the neuronal cell body to their sites of utilization at the axon terminus. To directly visualize this axonal transport in a living organism, we constructed transgenic lines of Caenorhabditis elegans that express green fluorescent protein fused to the monomeric synaptic vesicle transport motor, UNC-104. This UNC-104::GFP construct rescued the Unc-104 mutant phenotype and was expressed throughout the nervous system. Using time-lapse confocal fluorescence microscopy, we were able to visualize fluorescent motor proteins moving in both directions along neuronal processes, some of which were identified definitely as axons and others as dendrites. Using kymograph analysis, we followed the movement of $>900$ par- ticles. Most of them moved in one direction, but not necessarily at the same velocity. Ten percent of the observed particles reversed direction of movement during the period of observation, and $10 \%$ exhibited periods of movement interspersed with pauses. During episodes of persistent movement, particles moved at an average velocity of $1.02 \mu \mathrm{m} / \mathrm{sec}$, which is close to the in vitro velocity of microtubule gliding driven by purified monomeric kinesin at high motor density. To our knowledge, this is the first direct visualization and analysis of the movement of specifically labeled microtubule motor proteins along axons in vivo.

Key words: monomeric kinesin; UNC-104; Caenorhabditis elegans; axonal transport; time-lapse confocal microscopy; in vivo motor movement
The neuron is a highly polarized cell that elaborates two processes, a dendrite, specialized for neuronal signal reception, and an axon, specialized for neuronal signal conduction and transmission. The formation and function of both types of processes are thought to depend on the microtubule-based transport of cellular components from their sites of synthesis in the neuronal cell bodies to their sites of utilization in the axonal and dendritic termini (Goldstein and Yang, 2000). The nematode Caenorhabditis elegans is emerging as a useful system for studying intracellular transport events (Koushika and Nonet, 2000). C. elegans has a simple nervous system consisting of 302 neurons with structure and connectivity that are well characterized (White et al., 1986).

We previously developed a time-lapse fluorescence microscopy assay that allowed us to visualize specifically labeled motor proteins and their cargo molecules moving along dendrites and sensory cilia within chemosensory neurons in the head of $C$. elegans (Orozco et al., 1999; Signor et al., 1999). Here, for the first time, we have extended this analysis to the visualization of axonal transport in vivo by focusing on the monomeric kinesin UNC-104 in C. elegans.

The $u n c-104$ gene encodes a kinesin-like protein (Otsuka et al., 1991). Studies on UNC-104 and its mammalian ortholog, KIF1A,

Received Oct. 23, 2000; revised March 14, 2001; accepted March 19, 2001.

This work was supported by National Institutes of Health Grant GM50718 to J.M.S. We thank Dr. Andrew Fire for providing the GFP vectors, Dr. Yugi Kohara for the $y k 16 \mathrm{~g} 10 \mathrm{cDNA}$ clone, the C. elegans Genetic Center for the worm strains, and the Sanger Center for the cosmid clones. We also thank Dr. Lesilee Rose, Dr. Bo Liu, Dr. Tri Nguyen, and members of the Scholey laboratory for discussion and Kristine Adjemian for outstanding technical support.

Correspondence should be addressed to Dr. Jonathan M. Scholey, Section of Molecular and Cellular Biology, University of California at Davis, 1 Shields Avenue, Davis, CA 95616. E-mail: jmscholey@ucdavis.edu.

Copyright $\odot 2001$ Society for Neuroscience $0270-6474 / 01 / 213749-07 \$ 15.00 / 0$ showed that these are monomeric motors that move toward the plus ends of microtubules at 1.2-1.7 $\mu \mathrm{m} / \mathrm{sec}$ in vitro (Okada et al., 1995; Pierce et al., 1999). Mutations in the unc-104 gene in $C$. elegans result in uncoordinated, slow body motion and a slow growth rate. In the neurons of such mutants, the concentration of synaptic vesicles increases in cell bodies and decreases in synapses (Hall and Hedgecock, 1991). Therefore, it has been hypothesized that UNC-104 is a neuron-specific motor protein that is used for the anterograde axonal transport of synaptic vesicles.

To test the hypothesis that UNC-104 is an axonal motor protein, we generated transgenic lines of $C$. elegans expressing UNC-104 fused to green fluorescent protein (GFP) in a functional form. Using the in vivo transport assay, we were able to visualize the bidirectional movement of UNC-104::GFP along neuronal processes.

\section{MATERIALS AND METHODS}

Strains and growth of C. elegans. The wild-type strain N2 and strain CB1265 [unc-104 (e1265) II] were used. C. elegans were grown and maintained as described previously (Brenner, 1974).

Construction of UNC-104::GFP. The unc-104 gene and upstream regulatory sequences were subcloned into the pPD 95.77 GFP vector using standard molecular biology protocols (Maniatis et al., 1982). Specifically, a $2.3 \mathrm{~kb}$ SphI-Aat II unc-104 fragment (including exons 5-10) from cosmid C52E12 was cloned into pGEM-7Zf vector (Promega, Madison, WI) and named Part II. A $3.0 \mathrm{~kb}$ fragment representing the $3^{\prime}$ end of the unc-104 gene (exons 10-22) was PCR-amplified from the cDNA clone yk16g10 using the primers $5^{\prime}$-tatgctcaacaagaacttc- $3^{\prime}$ and $5^{\prime}$-caactgcagtgaagcagcaattgaagatg- $3^{\prime}$. The Pst $\mathrm{I}$ site introduced at the stop codon for the unc-104 gene is underlined. This $3.0 \mathrm{~kb}$ fragment was shuttled through the pGEM-T vector (Promega) and was named Part III. Ligating the 2.3 kb SphI-AatII from Part II with the $2.7 \mathrm{~kb}$ AatII-PstI fragment from Part III generated a $5.0 \mathrm{~kb}$ fragment. This $5.0 \mathrm{~kb}$ SphI-PstI fragment was cloned into the SphI-PstI site in pPD95.77 and named Part II+III. A 6.2 kb SphI-SphI fragment from cosmid C47A5 was characterized (data not 
shown) and found to overlap a fragment of cosmid C52E12. This SphI$S p h \mathrm{I}$ fragment extended from $3.7 \mathrm{~kb}$ upstream of the first exon to the intron between exons 4 and 5 of unc-104. The plasmid clone of UNC-104::GFP was constructed by inserting the $6.2 \mathrm{~kb} S p h \mathrm{I}-S p h \mathrm{I}$ fragment into Part II+III in the correct orientation.

Transformation of C. elegans. Heritable lines of transgenic worms carrying extrachromosomal arrays of the UNC-104::GFP construct were created by microinjection of the aforementioned plasmid UNC-104::GFP, with or without plasmid pRF4 containing the semidominant marker mutation rol-6 (su1006), into hermaphrodites by methods described previously (Fire, 1986; Kramer et al., 1990; Mello et al., 1991). After microinjecting UNC-104::GFP $(50 \mu \mathrm{g} / \mathrm{ml})$ and pRF4 $(50 \mu \mathrm{g} / \mathrm{ml})$ into wild-type N2 animals, we selected heritable roller lines (ejEx47-2, ejEx52-1, and ejEx51-1). To rescue the mutant phenotype, UNC-104::GFP $(70 \mu \mathrm{g} / \mathrm{ml})$ was microinjected into the unc-104 (e1265) animals, and rescued lines (ejEx72-1) were selected on the basis of their sinusoidal, wild-type movement. Growth rate was assayed by the brood size of a single worm in a $3 \mathrm{~d}$ period. Locomotion was assayed by touching the head or tail of the worm with an eyelash to stimulate backward or forward movement.

Expression pattern and in vivo transport assay. The expression and transport of UNC-104::GFP particles were analyzed by confocal microscopy. Worms expressing UNC-104::GFP were mounted on $2 \%$ agarose pads and anesthetized with $10 \mathrm{~mm}$ levamisole in M9 buffer. For analysis of the expression pattern, images were acquired on a Leica TCS NT confocal microscope with a $100 \times, 1.4$ numerical aperture (NA) objective. We acquired 16-60 focal planes and projected them to obtain the full pattern.

Neurons and ganglia were identified by comparing transmission and fluorescent images with the neuronal anatomy and connectivity diagrams described by White et al. (1986). The difference between axons and dendrites is not as clear as it is in mammalian nervous systems. When we could clearly see the process, we used its relative position to determine its connection pattern; we defined a process between a cell body and its final presynaptic terminal as an axon and a process between the cell body and the original postsynaptic terminal as a dendrite. Generally, we could identify axons and dendrites in the head, for example, the four sublateral nerves, which are axons, and the chemosensory dendrites, which are easily identified. The sublateral nerves terminate in the middle of the head, whereas the chemosensory dendrites terminate at the base of the cilia in the lips.

Transport was visualized by time-lapse confocal microscopy. Images were collected on an Olympus microscope equipped with an UltraView spinning disk confocal head (PerkinElmer Wallac Inc., Gaithersburg, MD) with a $100 \times, 1.4$ NA objective at a rate of three to eight frames per second. Images were analyzed using Metamorph Imaging software (Universal Imaging Corporation, West Chester, PA). A line was drawn over the process of interest, and the kymograph function was used to obtain an image of that line as a function of time. Particles appear as lines; for a moving particle, this line is oblique and its slope corresponds to the velocity of the particle. The lines obtained for stationary particles were used to correct for movement of the animal. Velocities were calculated for periods of persistent movement.

\section{RESULTS}

To analyze the expression and transport of the monomeric motor protein UNC-104, we created transgenic lines by microinjection of a transgene encoding an UNC-104::GFP fusion. This plasmid contained UNC-104 regulatory and coding sequences in frame with the GFP sequence. Three lines were generated by injection into wild-type N2 animals. ejEx47-2 and ejEx52-1 display normal roller behavior, and ejEx51-1 displays uncoordinated roller behavior. ejEx72-1 was generated by injection into unc-104 mutant animals and selection of rescued progeny with wild-type behavior.

\section{Rescue of unc-104 mutants}

When UNC-104::GFP was expressed in the unc-104 (e1265) mutant background, we observed rescue of both the growth rate (data not shown) and the locomotion of the animals (Fig. 1). In some cases, we observed complete rescue of the mutant phenotype, with the transgenic animals displaying normal sinusoidal

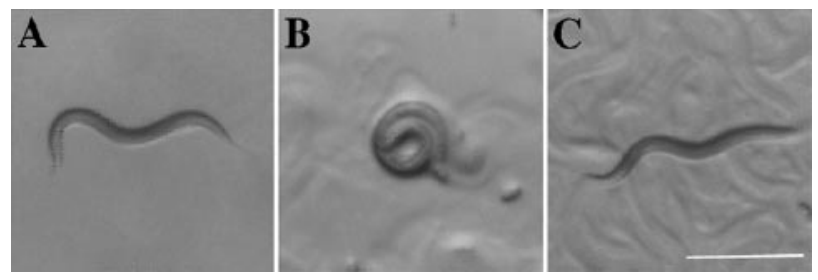

Figure 1. UNC-104::GFP rescues the unc-104 mutant (e1265). Snapshots of movement of adult worms under a dissecting scope are shown for a wild-type N2 worm $(A)$, a unc-104 (e1265) mutant $(B)$, and a rescued transgenic ejEx72-1 worm $(C)$. Animals were oriented with their heads on the left and their dorsal sides up. Note that N2 and ejEx72-1 extend their bodies and move in smooth, relatively linear trajectories, but the unc-104 (e1265) mutant curls up its body and displays paralytic phenotype. Scale bar, $0.5 \mathrm{~mm}$.

locomotion indistinguishable from wild-type animals. In other cases, the rescue was only partial; the worms displayed significantly improved forward or backward locomotion but showed differences in the speed of movement and the pattern of tracks when compared with wild type. For example, $52 \%$ of the progeny of one fully rescued $e j E x 72-1$ worm also showed complete rescue, but $38 \%$ of the progeny displayed only partial rescue, and the remaining $10 \%$ had the Unc-104 phenotype. The extent of rescue appeared to correlate with the level of expression of UNC-104::GFP as monitored by fluorescence intensity (see below). The observation that the UNC-104::GFP can rescue the mutant phenotype suggests that the fusion protein can carry out the normal functions of UNC-104.

\section{Phenotypic effects of UNC-104::GFP in wild-type animals}

Injection of the UNC-104::GFP construct into wild-type (N2) hermaphrodites resulted in variable phenotypes in $<5 \%$ of the progeny. One line (ejEx51-1) was characterized by the appearance of dumpy, small adults that displayed uncoordinated movement. This phenotype was similar to that caused by unc-104 mutant alleles (Hall and Hedgecock, 1991). As monitored by fluorescence intensity, UNC-104::GFP was expressed in these animals, suggesting that expression of extra transgenic protein can sometimes interfere with the normal transport of synaptic vesicles driven by UNC-104.

\section{Expression pattern of UNC-104::GFP}

We studied the expression pattern of UNC-104::GFP in both wild-type and unc-104 mutant backgrounds. The expression pattern was essentially identical (Fig. 2). UNC-104::GFP was expressed consistently throughout the nervous system. Distinct fluorescence was observed in neurons in the head (Fig. $2 A$ ), in the nerve ring (Fig. 2B), in the nerve cords (Fig. 2C,E), around the vulva (Fig. $2 D$ ), and in the tail (Fig. $2 F$ ), consistent with the hypothesis that UNC-104 is a neuronal transport motor. UNC-104::GFP was found in neuronal processes and the cytoplasm, but not in nuclei (Fig. 3). In some cases, the neuronal processes could be classified into either axons or dendrites, but there were no recognizable differences in UNC-104 expression between axons and dendrites. UNC-104::GFP was expressed during most stages of development, from late embryo to adult.

Although the overall expression pattern was similar in all transgenic animals that we examined, we observed variability in the actual amount of fluorescent protein that was expressed. For example, transgenic animals that displayed complete rescue of the Unc-104 phenotype expressed UNC-104::GFP in more neurons 

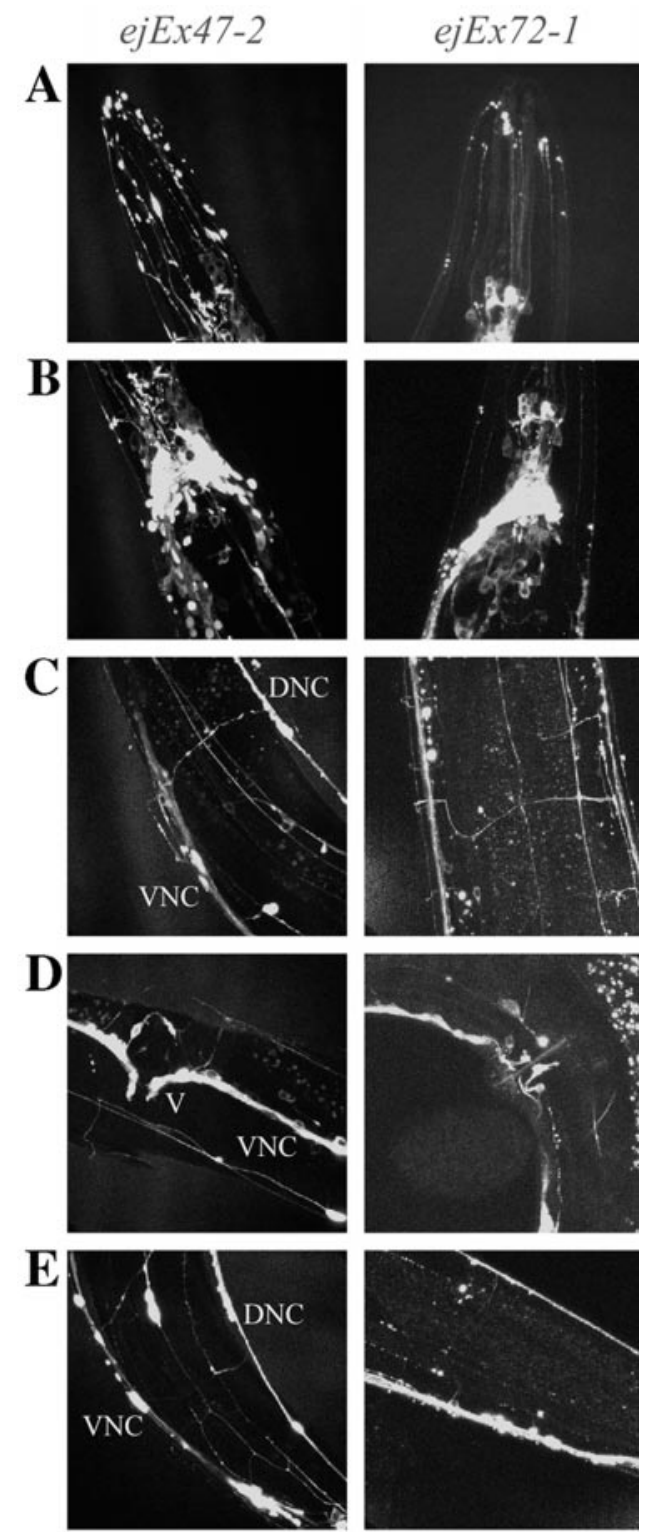

$\mathbf{F}$
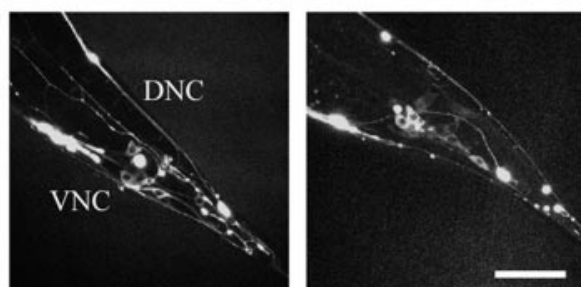

Figure 2. The expression pattern of UNC-104::GFP was studied in wild-type (ejEx47-2) and rescued mutant (ejEx72-1) worms. Images from various regions of the body are shown. $A$, Head; $B$, nerve ring; $C$, ventral and dorsal nerve cords between nerve ring and vulva; $D$, ventral nerve cord at vulva; $E$, ventral and dorsal nerve cords between vulva and tail; and $F$, tail. All images were projected and oriented with the animal's head facing either upward or to the left and the ventral side pointing to the left or downward. $V N C$, Ventral nerve cord; $D N C$, dorsal nerve cord; $V$, vulva. Scale bar, $25 \mu \mathrm{m}$.

and at a higher level than those that displayed only partial rescue. The wide distribution of UNC-104::GFP within the nervous system suggests that UNC-104 is present in most neuronal processes, including axons and dendrites.
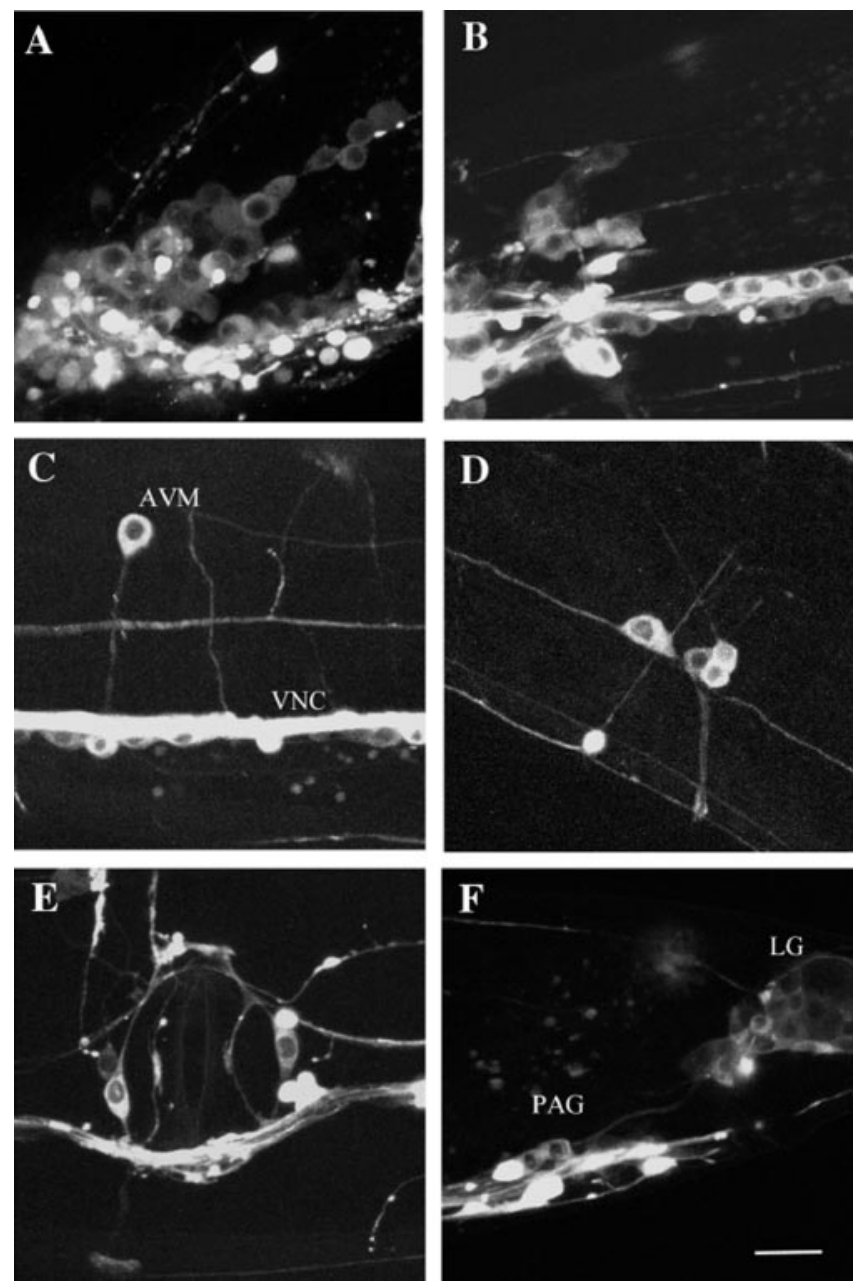

Figure 3. High magnification images showing expression pattern of UNC-104::GFP in a wild-type transgenic worm ejEx52-1. Animals were oriented with their heads on the left side and their dorsal sides facing up. Scale bar, $10 \mu \mathrm{m}$. $A$, Left side of the worm showing the lateral and ventral ganglion; $B$, retrovesicular ganglion; $C$, AVM neuron and ventral nerve cord $(V N C) ; D$, left posterior lateral ganglion; $E$, vulva; $F$, preanal ganglion $(P A G)$ and lumbar ganglion $(L G)$.

\section{Transport of UNC-104::GFP in neuronal processes}

To visualize the movement of fluorescently labeled UNC-104 motors along neuronal processes, we selected transgenic worms that displayed full rescue of the Unc-104 phenotype, that is, worms that exhibited coordinated locomotion. The fluorescent fusion protein is likely to be fully functional in these worms.

Time-lapse examination of neuronal processes in these animals revealed movement of the fluorescent fusion protein (Figs. 4, 5). The movement of bright particles could easily be followed from frame to frame (Fig. 4A), and we could manually obtain the velocity of the particle by finding its position in every frame. A kymograph gives a picture of the distance moved as a function of time, with moving particles appearing as oblique lines above the background (Fig. 4B). The slope of this line corresponds to the velocity of the particle and was the same as that obtained by manually tracking the particle. Kymographs were more efficient and more sensitive, because they allowed us to visualize dim particles and to follow many particles on one process.

Particles moved along different types of processes, including axons and dendrites, with similar velocities (Table 1). Generally, 
A

Figure 4. Transport of a UNC-104::GFP particle in a neurite. $A$, Individual images from time-lapse recording. The animal was oriented with its head to the left and its ventral side facing down. The neurite in which transport was observed was parallel to the ALML axon. The arrowhead shows the starting point, and the arrow points to the position of the moving particle. The open arrow indicates the start and direction of the process for the kymograph. The focal plane was changed during the recording to follow the process. Scale bar, $10 \mu \mathrm{m}$. $B$, Kymograph for process in $A$. The horizontal open arrow represents $10 \mu \mathrm{m}$. The vertical solid arrow represents $20 \mathrm{sec}$. Images for video (supplementary information, http:// www.mcb.ucdavis.edu/faculty-labs/scholey/ unc-104.html) were captured at $0.264 \mathrm{sec}$ intervals.

Figure 5. Transport of UNC-104::GFP particles. $A$ shows the anterior ventral sublateral axonal processes from SAAVL and SABVL neurons, $B$ shows a neurite process close to the left seam thread and lateral process in the middle of the body, and $C$ shows another neurite process. Animals were oriented with their heads to the left and their vulva facing down. Scale bars, 10 $\mu \mathrm{m}$. The arrow indicates the start and direction of the process recorded in the kymographs. $A^{\prime}, B^{\prime}, C^{\prime}$, Kymographs for processes in $A, B$, and $C$. The horizontal arrow represents $10 \mu \mathrm{m}$, and the vertical arrow represents $20 \mathrm{sec} . A^{\prime \prime}, B^{\prime \prime}, C^{\prime \prime}$, Drawings of some of the tracks in the kymographs. Images for supplementary videos (http:// www.mcb.ucdavis.edu/faculty-labs/scholey/ unc-104.html) were captured at intervals of $0.328,0.287$, and $0.309 \mathrm{sec}$, respectively.

A

B

C
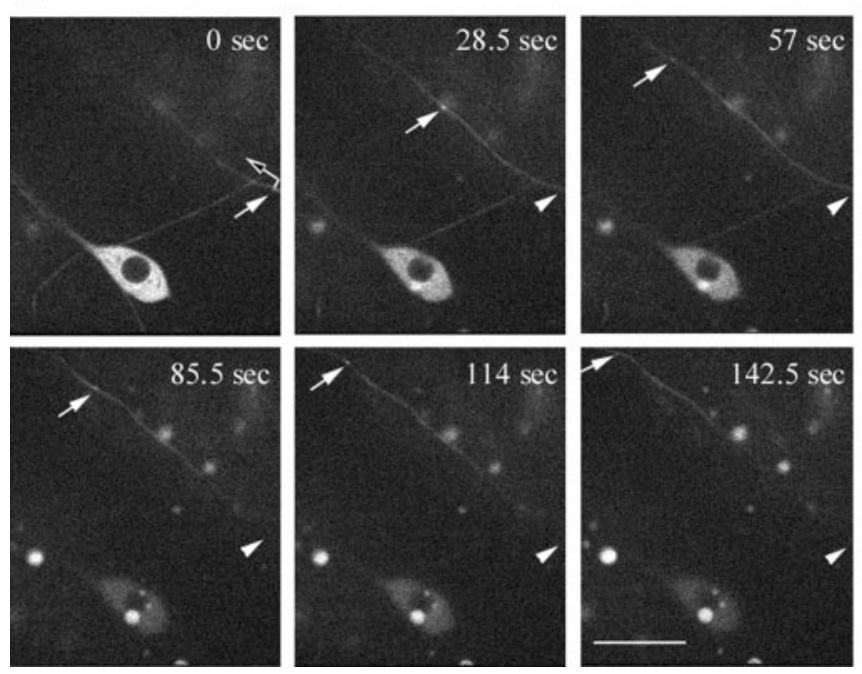

B
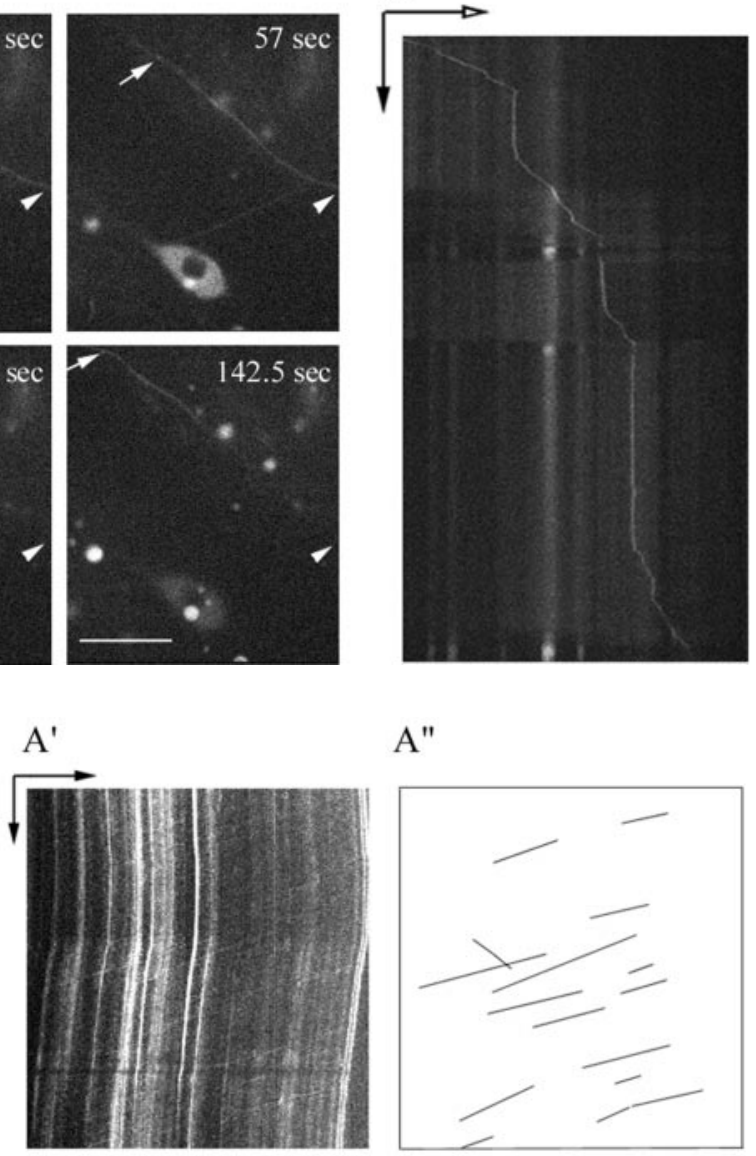

A"

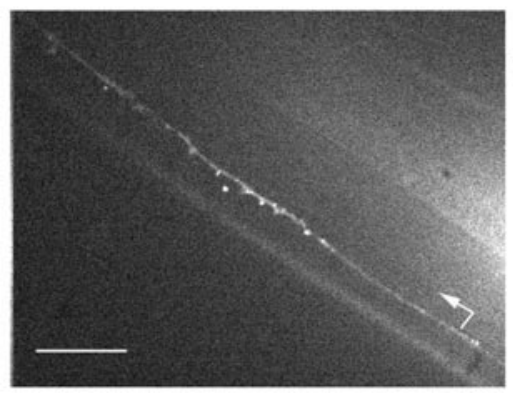

B'
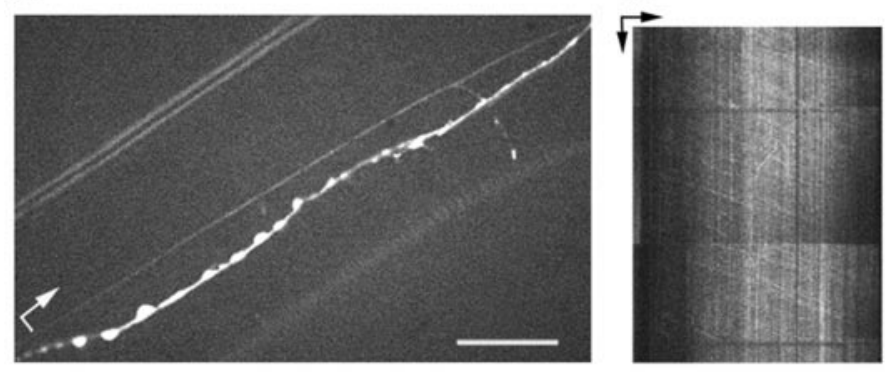

B"
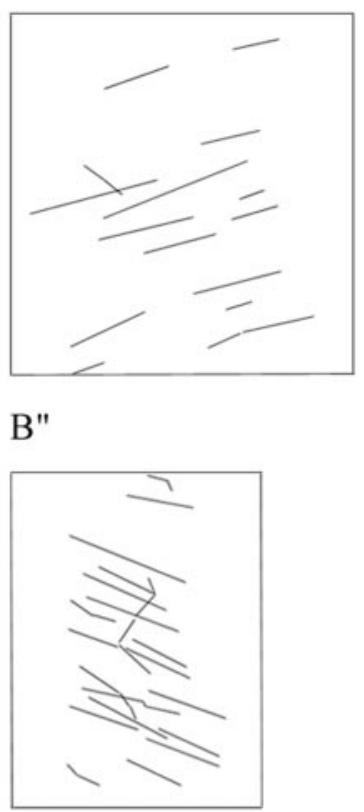

$\mathrm{C}^{\prime}$

C"
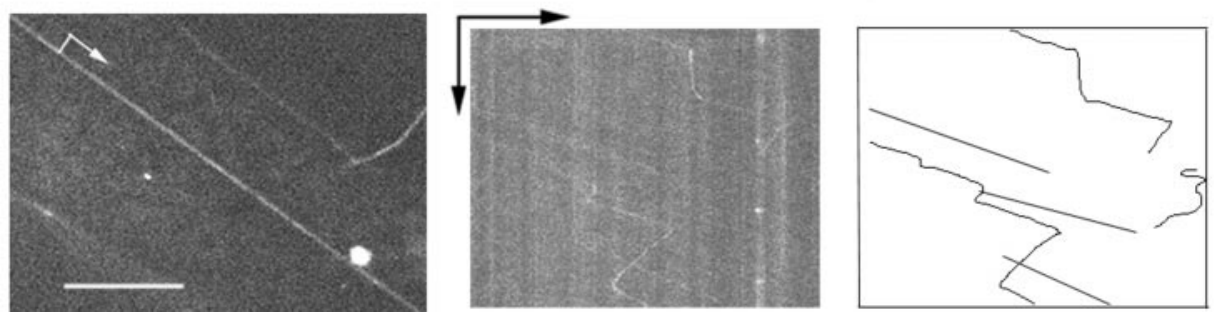

particles moved in one direction, sometimes changing velocity. In some processes, many particles moved at about the same velocity, as shown by the number of parallel lines in the kymograph (Fig. $5 A, B)$. Approximately $10 \%$ of the observed particles exhibited saltatory movement with periods of movement interspersed with pauses (Fig. 4, Table 2), and $\sim 10 \%$ of the particles reversed their direction of motion (Fig. 5C, Table 2). The velocity distribution of all observed particles is unimodal (Fig. 6), with a mean velocity during periods of persistent movement of $1.02 \mu \mathrm{m} / \mathrm{sec}$ (Table 1). The duration of uniform transport varied between 0.5 and $40 \mathrm{sec}$.

In some cases, we were able to unambiguously identify the observed process as an axon (Fig. $5 A$ ) or a dendrite. Movement in identified axons and dendrites occurred both anterogradely and retrogradely, but we observed about twice as many movements in 
Table 1. Transport properties of UNC-104:: GFP particles

\begin{tabular}{lllc}
$\begin{array}{l}\text { Type of process } \\
\text { (number of particles) }\end{array}$ & $\begin{array}{l}\text { Average velocity } \\
(\mu \mathrm{m} / \mathrm{sec})\end{array}$ & $\begin{array}{l}\text { Persistence of } \\
\text { movement at } \\
\text { uniform } \\
\text { velocity }(\mathrm{sec})\end{array}$ & $\begin{array}{l}\text { Number } \\
\text { of events }\end{array}$ \\
\hline Axon (385) & Ant $1.01 \pm 0.53$ & $4.89 \pm 3.84$ & 464 \\
& Ret $1.06 \pm 0.58$ & $4.68 \pm 4.56$ & 237 \\
Dendrite (37) & Ant $1.19 \pm 0.38$ & $5.77 \pm 3.77$ & 50 \\
Axonal commissure (26) & Ret $0.98 \pm 0.48$ & $3.67 \pm 3.02$ & 24 \\
Unidentified commissure (221) & $1.03 \pm 0.37$ & $5.80 \pm 3.59$ & 33 \\
Unidentified process (248) & $0.98 \pm 0.51$ & $4.54 \pm 3.49$ & 362 \\
All particles (917) & $1.03 \pm 0.53$ & $6.81 \pm 5.80$ & 464 \\
\hline Paricis & $1.02 \pm 0.53$ & $5.35 \pm 4.60$ & 1634
\end{tabular}

Particle velocities were calculated during periods of uniform movement. The number of events is larger than the number of particles because particles sometimes changed velocities or direction or paused before resuming movement. Ant, Anterograde; Ret, retrograde.

the anterograde direction as in the retrograde direction (Table 1). The visualization of movement supports the hypothesis that UNC-104 is a transport motor protein in axonal processes and raises the possibility that it may also function in dendritic processes.

\section{DISCUSSION}

Here we report the production of transgenic lines of $C$. elegans expressing the monomeric kinesin, UNC-104, fused to green fluorescent protein. We observed UNC-104 expression and movement in all types of processes, including axons and dendrites. The functional significance of movement of UNC-104 along processes identified as dendrites is unclear at this point, but it could reflect a role for UNC-104 in dendrodendritric neurotransmission. However, our observation that UNC-104::GFP moves along axons is consistent with studies suggesting that UNC-104 functions as an axonal synaptic vesicle transport motor (see below).

Our observation that the UNC-104::GFP transgene rescued the Unc-104 phenotype suggests that it encodes a fusion protein capable of carrying out the normal functions of the UNC-104 motor, which include binding presynaptic vesicles and transporting them on microtubules using energy released from ATP hydrolysis. The GFP tag was added at the $\mathrm{C}$ terminus, where it is unlikely to interfere with the ATPase and microtubule motility activities of the N-terminal motor domain but it could potentially interfere with proper cargo binding. Although vesicle binding and the distribution of synaptic vesicles were not studied, the observed mutant rescue suggests that the UNC-104::GFP fusion protein must be binding its presynaptic vesicle cargo and transporting it along microtubules to its proper destination.

The injection of the UNC-104::GFP construct into wild-type animals sometimes resulted in a dominant negative phenotype that phenocopies the unc-104 mutant. Introduction of extra copies of the unc-104 gene could trigger gene silencing mechanisms like RNA interference (Grishok et al., 2000). However, UNC-104::GFP expression in these animals is very similar to that observed in normal transgenic animals, suggesting that the protein is translated and it is the introduction of the fusion protein that leads to the phenotype. One possible explanation is that the increase in the intracellular concentration of UNC-104 creates an organelle jam along the axon. This could happen if the excess motor exceeds the supply of a limiting accessory factor required for transport, which would lead to a surplus of inactive motors that could exert drag forces on the cargo or saturate the microtubule tracks and inhibit normal transport. Alternatively, increasing the supply of active anterograde motors could override the corresponding retrograde transport system and interfere with the recycling of essential components back to the cell body, which could phenocopy mutants in the anterograde transport pathway. For example, mutations in the retrograde intraflagellar transport motor, che-3-dynein, cause ciliary and chemosensory defects that phenocopy mutations in the corresponding anterograde pathway in $C$. elegans (Perkins et al., 1986; Signor et al., 1999; Wicks et al., 2000). We also note that the tail domain of conventional kinesin is an inhibitory regulator of the motor domain (Coy et al., 1999; Friedman and Vale, 1999; Hackney and Stock, 2000). If this is the case for UNC-104, then addition of GFP could abolish this regulation,

Table 2. Particle pauses and changes in direction

\begin{tabular}{lllll} 
Type of process & $\begin{array}{l}\text { Total } \\
\text { number of } \\
\text { particles }\end{array}$ & $\begin{array}{l}\text { Number of } \\
\text { particles that } \\
\text { change direction }\end{array}$ & $\begin{array}{l}\text { Number of } \\
\text { particles that } \\
\text { pause }\end{array}$ & $\begin{array}{l}\text { Average pause du- } \\
\text { ration (number) }\end{array}$ \\
\hline Axon & 385 & 30 & 39 & $6.20 \pm 7.71 \mathrm{sec}(62)$ \\
Dendrite & 37 & 1 & 3 & $3.46 \pm 2.58 \mathrm{sec}(4)$ \\
Commissure & 247 & 30 & 23 & $4.06 \pm 3.52 \mathrm{sec}(34)$ \\
Unidentified process & 248 & 33 & 34 & $5.02 \pm 6.21 \mathrm{sec}(60)$
\end{tabular}

The number of particles that changed direction and the number of particles that exhibited pauses were quantitated on each type of process. The duration of pauses varies widely. The number of pauses is larger than the number of particles, because a single particle could pause repeatedly. The data in both tables were derived from observations in the four anterior sublateral processes, the sensory nerves in the head and the tail, sublateral and lateral processes, the dorsal and ventral nerve cords, processes from the ALM and AVM neurons, and the commissures. 


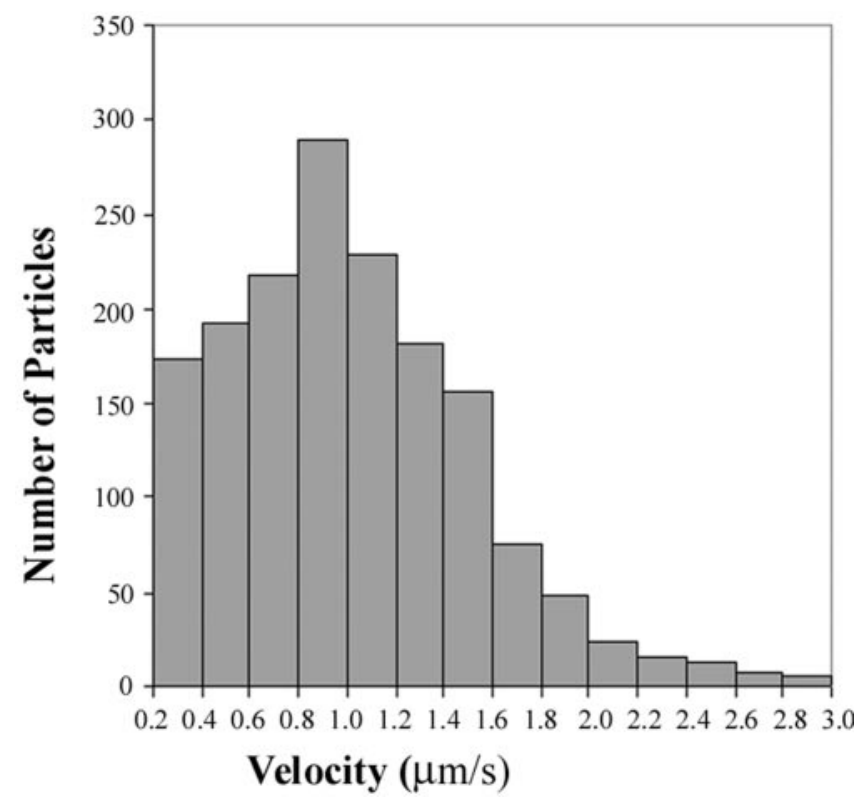

Figure 6. Histogram of transport velocities. The number of particles moving at given velocity ranges is shown; velocities $<0.2 \mu \mathrm{m} / \mathrm{sec}$ were considered as pauses and were not included. The histogram is unimodal.

leading to motors that are constitutively active in ATP hydrolysis and motility, which could give rise to ATP depletion. Such a depletion of energy stores could conceivably contribute to the observed uncoordinated phenotype.

Accumulation of synaptic vesicles in the cell bodies of unc-104 mutants (Hall and Hedgecock, 1991) suggests that UNC-104 is expressed throughout the nervous system. In accordance with this hypothesis, our direct examination of the expression pattern, which has not been studied before, revealed that UNC-104::GFP fluorescence was distributed throughout most neuronal processes, including axons and dendrites, and cell bodies. Our results are also consistent with the report that the presumptive cargo protein, synaptobrevin (SNB-1), is expressed in all neurons in $C$. elegans (Nonet, 1999). SNB-1 is a transmembrane protein in synaptic vesicles, and synaptobrevin in mammalian cells is a cargo of KIF1A (Okada et al., 1995). It will be interesting to study the movement of both UNC-104 and SNB-1 (or other possible cargoes) simultaneously.

We observed movement of UNC-104::GFP along neuronal processes. The use of kymographs allowed us to follow the movement of many dim particles on one process, which often appear as a streaming background in the time-lapse movie. We measured an average velocity of $1.02 \mu \mathrm{m} / \mathrm{sec}$ for periods of persistent movement. This average velocity was obtained when images were acquired at rates of at least three frames per second. At the lower acquisition rates that we used in preliminary studies, fast velocities were only observed sometimes, and the velocity had a one-tailed distribution, leading to a biased, low-velocity average (data not shown). At fast acquisition rates, such as those used here, all moving particles could be tracked, and the histogram shows a peak velocity coincident with the calculated average velocity (Fig. 6).

The average velocity for periods of movement $(1.02 \mu \mathrm{m} / \mathrm{sec})$ is close to that measured in vitro in multiple motor assays (1.2 $\mu \mathrm{m} / \mathrm{sec}$, Okada et al., 1995; $1.7 \mu \mathrm{m} / \mathrm{sec}$, Pierce et al., 1999), consistent with the hypothesis that multiple UNC-104 motors transport a synaptic vesicle. It seems reasonable to propose that the observed difference (1.02 vs $1.2-1.7 \mu \mathrm{m} / \mathrm{sec})$ is not biologically significant and simply reflects differences in experimental conditions. However, we cannot rule out the possibility that this difference is significant. Motor movement may be slower in vivo than in vitro for several reasons. In vivo, motors have to bind to cargo, and there may be viscosity constraints along the process. A vesicle could have different types of motors bound moving at different speeds and/or in opposite directions. Pigment granules purified from Xenopus melanophores were found to have both plus-end and minus-end directed motors. One kind of motor predominated, but the opposing motor had some activity (Reese and Haimo, 2000). The final direction and the net velocity of transport would thus depend on the balance of forces created by all the motors, which would explain the occurrence of different velocities. Dissociation and association of motors from and to vesicles could also affect this balance and thus influence the net velocity and even the direction of movement.

In our assay, the movement of some particles was saltatory, with periods of movement interspersed with pauses. In a single motor assay, the movement of a monomeric construct containing the motor domain of KIF1A also appeared oscillatory, and motors sometimes paused or moved backward for a short distance (Okada and Hirokawa, 1999). Although we observed similar behavior, it is important to note that in our in vivo assay the motor moved at least eight times faster, suggesting that the two types of motility are not similar. As explained above, changes in the net balance of motor forces could change the velocity of a particle, and pauses are seen when the net force is zero. The discontinuity of microtubule tracks could also explain pauses if a motor at the end of one microtubule pauses before attaching to the next microtubule and continuing its movement.

In processes that were identified as axons, we observed bidirectional movement of UNC-104. We did not find a significant difference between the velocities in the anterograde and retrograde directions, but we did see twice as many particles moving in the anterograde direction as in the retrograde direction. There are several possible explanations for this bidirectional transport. In mammalian cells, axonal microtubules are all oriented with their plus end distal (Baas, 1999; Sharp et al., 2000). If this is the case in C. elegans, then UNC-104 could act as a transport motor in the anterograde direction but would have to be carried back in the retrograde direction by a minus end-directed motor in a "shuttle system" similar to that described for the kinesin-II transport pathway in chemosensory neurons of C. elegans (Signor et al., 1999). For example, UNC-104 could move actively in the anterograde direction and be moved passively by the same retrograde motors that recycle kinesin-II in chemosensory neurons (Signor et al., 1999). Because the latter pathway involves retrograde motors moving at $1.1 \mu \mathrm{m} / \mathrm{sec}$, this would result in bidirectional movement of UNC-104::GFP at a single velocity of $1.0-1.1 \mu \mathrm{m} / \mathrm{sec}$ as we observed. However, if microtubules are not of uniform polarity in axons of C. elegans, then UNC-104 could transport cargo in both directions by walking on microtubules of opposite orientations. It is therefore important to determine the polarity of microtubules in C. elegans neuronal processes to distinguish between these possibilities. Because we only observed transport of GFP, it is possible that the UNC-104::GFP transgene product was being carried as a cargo in both directions by other motors. However, the fact that it rescued the mutant phenotype argues that it is a functional 
motor and is carrying vesicles at least in the anterograde direction.

In conclusion, the data described here documents the first visualization of a specifically labeled motor moving along axons in a living animal. Together with other studies that described motor proteins moving along dendrites and sensory cilia within chemosensory neurons (Orozco et al., 1999; Signor et al., 1999), this work provides a useful basis for further studies of various forms of microtubule-based transport in neurons and other cell types within living $C$. elegans (Koushika and Nonet, 2000). The studies described here show that UNC-104::GFP fusion proteins are capable of carrying out the functions of wild-type UNC-104 proteins and that UNC-104::GFP punctae are capable of moving at velocities similar to those predicted from in vitro multiple motor motility assays. Our studies support the hypothesis that UNC-104 functions as a transport motor in axons and raise the possibility that it may function in other types of neuronal processes as well.

\section{REFERENCES}

Baas PW (1999) Microtubules and neuronal polarity: lessons from mitosis. Neuron 22:23-31.

Brenner S (1974) The genetics of Caenorhabditis elegans. Genetics 77:71-94.

Coy DL, Hancock WO, Wagenback M, Howard J (1999) Kinesin's tail domain is an inhibitory regulator of the motor domain. Nature Cell Biol 1:288-292.

Fire A (1986) Integrative transformation of Caenorhabditis elegans. EMBO J 5:2673-2680.

Friedman DS, Vale RD (1999) Single-molecule analysis of kinesin motility reveals regulation by the cargo-binding tail domain. Nat Cell Biol 1:293-297.

Goldstein LS, Yang Z (2000) Microtubule-based transport systems in neurons: the roles of kinesins and dyneins. Annu Rev Neurosci 23:39-71

Grishok A, Tabara H, Mello CC (2000) Genetic requirements for inheritance of RNA $\mathrm{R}_{\mathrm{i}}$ in $C$. elegans. Science 287:2494-2497.

Hackney DD, Stock MF (2000) Kinesin's IAK tail domain inhibits initial microtubule-stimulated ADP release. Nat Cell Biol 2:257-260.

Hall DH, Hedgecock EM (1991) Kinesin-related gene unc-104 is required for axonal transport of synaptic vesicles in C. elegans. Cell 65:837-847.
Koushika SP, Nonet ML (2000) Sorting and transport in C. elegans: a model system with a sequenced genome. Curr Opin Cell Biol 12:517-523.

Kramer JM, French RP, Park EC, Johnson JJ (1990) The Caenorhabditis elegans rol-6 gene, which interacts with the sqt-1 collagen gene to determine organismal morphology, encodes a collagen. Mol Cell Biol 10:2081-2089.

Maniatis T, Sambrook J, Fritsch EF (1982) Molecular cloning. Cold Spring Harbor, NY: Cold Spring Harbor Laboratory.

Mello CC, Kramer JM, Stinchcomb D, Ambros V (1991) Efficient gene transfer in C.elegans: extrachromosomal maintenance and integration of transforming sequences. EMBO J 10:3959-3970.

Nonet ML (1999) Visualization of synaptic specializations in live $C$. elegans with synaptic vesicle protein-GFP fusions. J Neurosci Methods 89:33-40.

Okada Y, Hirokawa N (1999) A processive single-headed motor: kinesin superfamily protein KIF1A. Science 283:1152-1157.

Okada Y, Yamazaki H, Sekine-Aizawa Y, Hirokawa N (1995) The neuron-specific kinesin superfamily protein KIF1A is a unique monomeric motor for anterograde axonal transport of synaptic vesicle precursors. Cell 81:769-780.

Orozco JT, Wedaman KP, Signor D, Brown H, Rose L, Scholey JM (1999) Movement of motor and cargo along cilia. Nature 398:674.

Otsuka AJ, Jeyaprakash A, García-Añoveros J, Tang LZ, Fisk G, Hartshorne T, Franco R, Born T (1991) The C. elegans unc-104 gene encodes a putative kinesin heavy chain-like protein. Neuron 6:113-122.

Perkins LA, Hedgecock EM, Thomson JN, Culotti JG (1986) Mutant sensory cilia in the nematode Caenorhabditis elegans. Dev Biol 117:456-487.

Pierce DW, Hom-Booher N, Otsuka AJ, Vale RD (1999) Singlemolecule behavior of monomeric and heteromeric kinesins. Biochemistry 38:5412-5421.

Reese EL, Haimo LT (2000) Dynein, dynactin, and kinesin II's interaction with microtubules is regulated during bidirectional organelle transport. J Cell Biol 151:155-165.

Sharp DJ, Rogers GC, Scholey JM (2000) Roles of motor proteins in building microtubule-based structures: a basic principle of cellular design. Biochim Biophys Acta 1496:128-141.

Signor D, Wedaman KP, Orozco JT, Dwyer ND, Bargmann CI, Rose LS, Scholey JM (1999) Role of a class DHC1b dynein in retrograde transport of IFT motors and IFT raft particles along cilia, but not dendrites, in chemosensory neurons of living Caenorhabditis elegans. J Cell Biol 147:519-530.

White JG, Southgate E, Thomson JN, Brenner S (1986) The structure of the nervous system of the nematode Caenorhabditis elegans. Philos Trans R Soc Lond B Biol Sci 314:1-340.

Wicks SR, de Vries CJ, van Luenen HGAM, Plasterk RHA (2000) $\mathrm{CHE}-3$, a cytosolic dynein heavy chain, is required for sensory cilia structure and function in Caenorhabditis elegans. Dev Biol 221:295307. 